\title{
Tamm plasmons in metal/nanoporous GaN distributed Bragg reflector cavities for active and passive optoelectronics
}

\author{
G. LheureuX,1,2,5,6 (D) M. Monavarian, ${ }^{1,2,5,7}$ (D) R. \\ Anderson, ${ }^{1,2,5}$ R. A. DeCRESCent, ${ }^{3}$ J. Bellessa, ${ }^{4}$ C. \\ SYMONDS, ${ }^{4}$ J. A. SCHULLER, ${ }^{3}$ J. S. SPECK, ${ }^{1,2}$ S. NAKAMURA, ${ }^{1,2}$ \\ AND S. P. DENBAARS ${ }^{1,2}$ \\ ${ }^{1}$ Materials Department, University of California Santa Barbara, Santa Barbara, California 93106, USA \\ ${ }^{2}$ Solid-State Lighting Energy Electronic Center (SSLEEC), University of California Santa Barbara, Santa \\ Barbara, California 93106, USA \\ ${ }^{3}$ Department of Physics, University of California Santa Barbara, Santa Barbara, California 93106, USA \\ ${ }^{4}$ Université Lyon, Université Claude Bernard Lyon 1, CNRS, Institut Lumière Matière, F-69622 Lyon, \\ France \\ ${ }^{5}$ These authors contributed equally to this work \\ 6glheureux@ucsb.edu \\ ${ }^{7}$ mmonavarian@ucsb.edu
}

\begin{abstract}
We theoretically and experimentally investigate Tamm plasmon (TP) modes in a metal/semiconductor distributed Bragg reflector (DBR) interface. A thin Ag (silver) layer with a thickness (55 nm from simulation) that is optimized to guarantee a low reflectivity at the resonance was deposited on nanoporous GaN DBRs fabricated using electrochemical (EC) etching on freestanding semipolar (20) $\overline{21}$ GaN substrates. The reflectivity spectra of the DBRs are compared before and after the $\mathrm{Ag}$ deposition and with that of a blanket $\mathrm{Ag}$ layer deposited on $\mathrm{GaN}$. The experimental results indicate the presence of a TP mode at $\sim 454 \mathrm{~nm}$ on the structure after the $\mathrm{Ag}$ deposition, which is also supported by theoretical calculations using a transfer-matrix algorithm. The results from mode dispersion with energy-momentum reflectance spectroscopy measurements also support the presence of a TP mode at the metal-nanoporous GaN DBR interface. An active medium can also be accommodated within the mode for optoelectronics and photonics. Moreover, the simulation results predict a sensitivity of the TP mode wavelength to the ambient ( $\sim$ 4-7 nm shift when changing the ambient within the pores from air with $n=1$ to isopropanol $n=1.3$ ), suggesting an application of the nanoporous GaN-based TP structure for optical sensing.
\end{abstract}

(C) 2020 Optical Society of America under the terms of the OSA Open Access Publishing Agreement

\section{Introduction}

A Tamm plasmon (TP) mode is an optical state that exists at the interface between a metallic layer and a distributed Bragg reflector (DBR). Named in analogy to the Tamm state [1], a class of surface states, TP modes were first experimentally demonstrated by Sasin et al. in 2008 [2] shortly after its theoretical prediction in 2006-2007 [3,4]. TP modes behave as "zero dimensional" (0D) cavity modes with a strong vertical confinement, resulting in a high quality factor over 1000 [5]. In contrast to other optical surface states associated with metals, TP modes can be either TEor TM-polarized and their dispersion relations exist within the light cone. Hence, TP modes can be directly coupled to light modes or surface plasmons [6], which makes them extremely interesting for optoelectronic applications.

TP-based Lasers [7-9], single photon emitters [10] and sensors [11] have recently been demonstrated. One of the most interesting benefits of TP modes is that their properties can 
be largely tuned simply by tailoring the metal component without the need for modifying the complete structure [12,13]. Historically used in arsenide material systems, TP structures have recently been explored in III-nitride systems, mainly to improve the light-extraction efficiency (LEE) of light-emitting diodes (LEDs) emitting at green wavelengths [14]. TP modes have also been realized in dielectric mesoporous DBRs [11], while the dielectric DBRs cannot be easily implemented in semiconductor-based optoelectronic and photonic platforms due to the epitaxial challenges.

The DBR mirrors used in TP structures can be constructed from dielectric or semiconductor materials. Dielectric DBRs typically provide high refractive index contrast between the layers, but may not be easily integrated into semiconductor technology due to epitaxial difficulties [15]. On the other hand, semiconductor DBRs suffer from low refractive index contrast and typically large lattice mismatch between the layers, particularly for nitride systems [16]. Ternary strain-compensated InAlN lattice-matched to GaN have been used as DBRs for lasers [17]. However, the growth of strain-compensated ternary DBRs requires complicated growth conditions. Also, due to the small refractive index contrast between $\mathrm{GaN}$ and its lattice-matched III-nitride alloy $\left(\operatorname{In}_{0.18} \mathrm{Al}_{0.82} \mathrm{~N}\right)$, normally a large number of pairs $(\sim 40$ or more, equivalent to $>8$ hours of growth time) is required to achieve a high reflectance $[18,19]$. Nanoporous $\mathrm{GaN}$ has been proposed as an alternative DBR material since it provides perfect lattice matching with refractive index contrasts as large as dielectric DBRs [20,21]. In addition, the porosity of nanoporous GaN can be tuned by doping and EC etching conditions [21] (the size and density of the pores are directly correlated with the bias voltage and doping density, respectively [20]). Therefore, the technology may provide access to a long-range of wavelengths for various applications [22-24]. Contrary to dielectric mesoporous DBRs, nanoporous GaN DBRs allow for the realization of active devices that can easily be integrated with industrial GaN processes.

In this work, we demonstrate the first observation of a TP mode appearing at the interface between nanoporous GaN DBR and a thin silver (Ag) layer. First, we theoretically calculate the TP mode wavelengths and demonstrate their strong dependence on the Ag layer thickness and DBR properties (such as porosity, number of pairs, and ambient within the pores). Experimentally, a steep dip in the DBR reflectivity spectrum after Ag deposition clearly indicates the presence of a TP mode at $\sim 454 \mathrm{~nm}$. Our simulation results indicate a $\sim 4-7 \mathrm{~nm}$ shift of the TP mode position when the nanoporous layer refractive index changes from 1.0 to 1.3 , indicating a strong potential of such structures for optical sensing applications. The nanoporous GaN-based TP modes can be easily integrated into optoelectronic and photonic technologies. The results suggest using nanoporous GaN-based TP structures for active (e.g. LEDs, lasers, etc.) and passive (e.g. optical sensors) applications and beyond.

\section{Theoretical calculations}

The TP dispersion and DBR reflectivity were calculated using a transfer-matrix algorithm. The optical index of the silver layer is obtained using a polynomial fitting of the experimental values obtained by Johnson and Christies [25]. The optical index $n_{\text {low }}$ of the porous layer is given by the volume average theory (VAT) as [21]

$$
n_{\text {low }}=\sqrt{(1-\rho) n_{G a N^{2}}+\rho n_{\text {por }}^{2}}
$$

where $\rho$ is the porosity of the layer, $n_{G a N}$ is the refractive index of GaN (chosen here as 2.36 [26]) and $n_{p o r}$ is the refractive index of the material inside the pore, normally considered as $n_{p o r}=1$ for air. In order to account for experimental fluctuations in the porosity, the porosity of the low index layers was randomly chosen, following a normal distribution with a mean of 53\% and standard deviation of $10 \%$. The applied fluctuations had a minimal impact on the final results. The central wavelength $\left(\lambda_{0}\right)$ of the DBR was chosen as $415 \mathrm{~nm}$ in the calculations. A slight shift 
in wavelength $(\sim 1 \mathrm{~nm})$ could be seen between different simulation batches due to the slight variations between different realizations of the randomly chosen porosity. However, the trend is not affected by the randomly chosen porosity in any way.

A way to see the particularity of the TP structure is to compute the electric field of a structure without and with the metallic layer. Figure 1 shows the simulated transverse electric (TE) mode electric field for a random nanoporous DBR without and with a $55 \mathrm{~nm}$ layer of silver on top. In the absence of the metallic layer [Fig. 1(a)], the natural mode of the nanoporous DBR are Braggs modeS or Bloch waves. The maximum of the field is located in the middle of the DBR. When a $55 \mathrm{~nm}$ of $\mathrm{Ag}$ is added on top of the DBR, a drastic change in the field distribution is visible [Fig. 1(b)]. The electric field peak amplitude exponentially decays from the metal/DBR interface to the bottom of the DBR. The maximum of the field is located within the first high-index layer of the DBR. This is a classic proof of the presence of a TP mode at the interface between the metal and the DBR. It is worth noting that the random distribution in the porosity (resulting in the random variation of the $n_{\text {low }}$ along the DBR) considered in the simulations is not detrimental to the formation of the TP mode, indicating the robustness of the TP structure design.
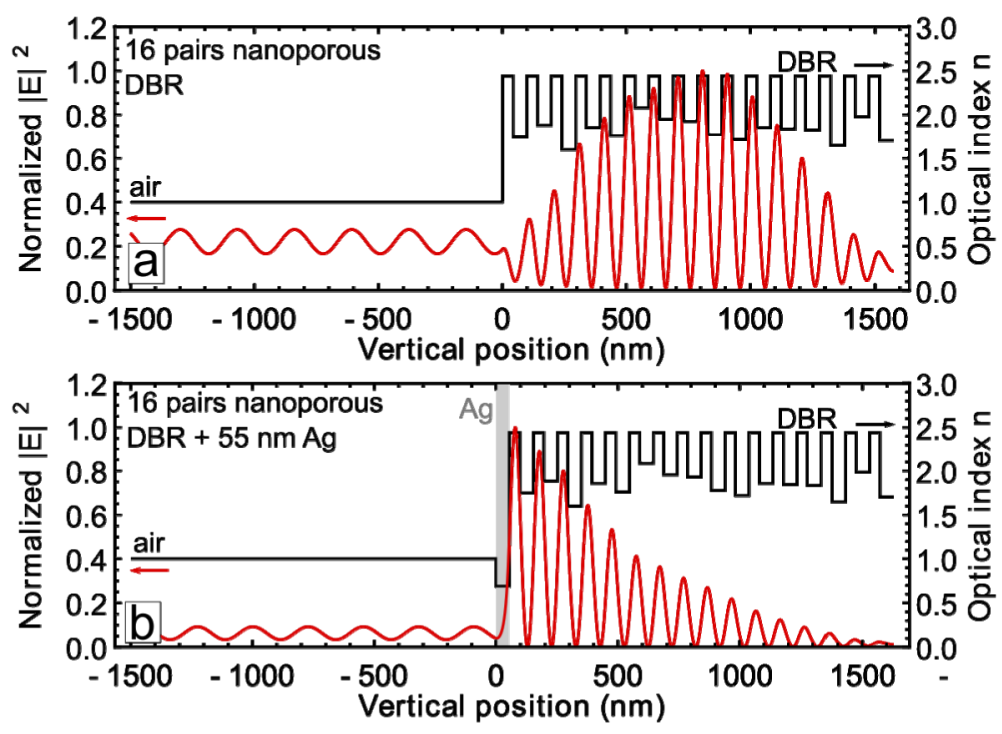

Fig. 1. Spatial repartition of the normalized squared electric field calculated for a) a Bragg mode $(\lambda=459 \mathrm{~nm})$ (top) and b) a Tamm plasmon mode $(\lambda=454 \mathrm{~nm})$ (bottom) in TE polarization.

As stated in the introduction, a TP structure can be seen as a cavity where the electric field is confined in the mirror. Figure 2(a) shows the reflectivity spectra of nanoporous GaN DBRs with varying number of pairs without [Fig. 2(a)] and with [Fig. 2(b)] a $55 \mathrm{~nm} \mathrm{Ag}$ layer on top. The peak reflectivity of the DBR increases by increasing the number of pairs, up to the point where the phase matching condition for the apparition of TP mode is realized ( $\sim 8$ pairs, based on the calculations) [4,27]. Increasing the number of pairs results in an improved quality factor and a slight blue shift of the mode. Increasing the number of pairs above 20, does not further improve the quality factor of the cavity and the TP mode position does not shift any further [Fig. 2(c)]. Since the difference between the TP modes for 16 and 20 pairs is insignificant, for the sake of simplicity a 16 pairs of DBR is considered for this study. In this work, we will only consider the case in which light is incident to the metal side of the structure, while previous works [27-29] have established that a TP mode can also be realized when the light is incident to the DBR side. 


\section{Optics EXPRESS}
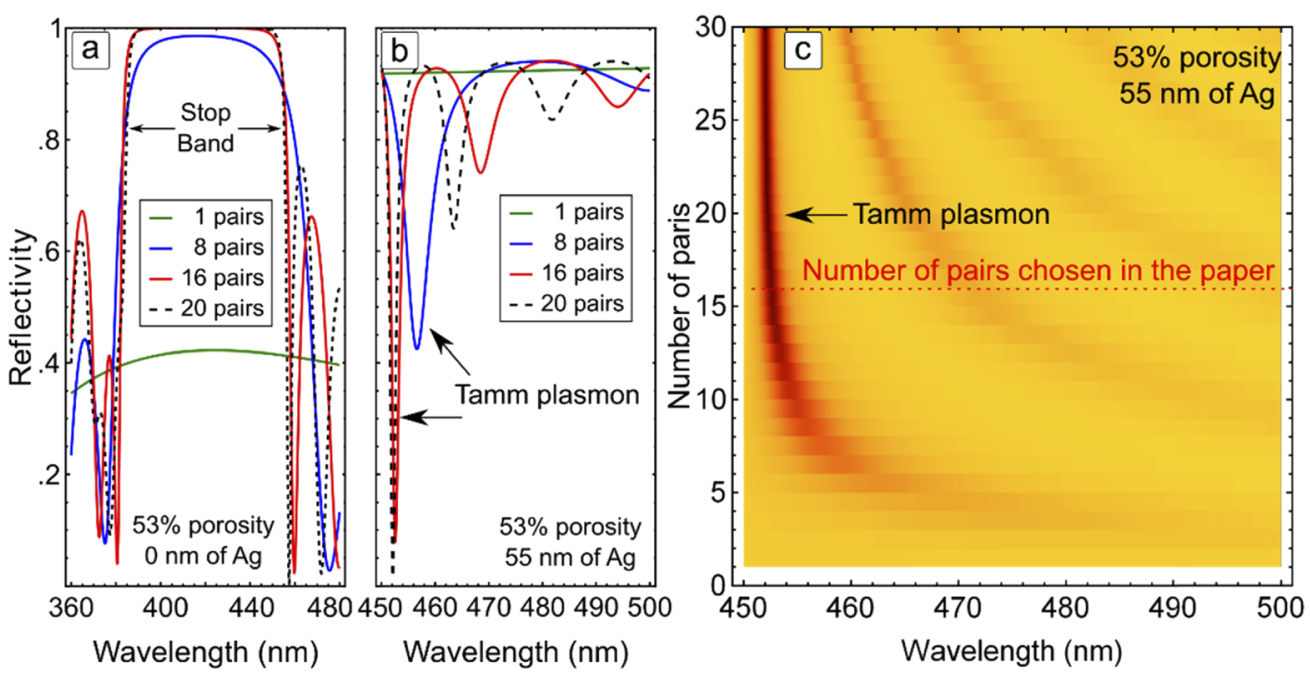

Fig. 2. Simulated reflectivity spectra of nanoporous DBR for various number of pairs (a) without and (b) with a $55 \mathrm{~nm} \mathrm{Ag} \mathrm{layer.} \mathrm{(c)} \mathrm{Density} \mathrm{plot} \mathrm{of} \mathrm{the} \mathrm{nanoporous} \mathrm{Tamm} \mathrm{plasmon}$ structure $(\mathrm{DBR}+\mathrm{Ag})$ with various number of pairs. The Ag thickness $(55 \mathrm{~nm})$ and the porosity $(53 \%)$ were kept constant.

Figure 3(a) shows the calculated normal incidence reflectivity spectrum of a 16-pair GaN/nanoporous GaN DBR without (dash black line) and with (solid red line) a $55 \mathrm{~nm} \mathrm{Ag} \mathrm{layer.} \mathrm{A} \mathrm{sharp} \mathrm{reflectivity}$ minima within the stop band of the DBR is observed, indicative of a TP mode. The dispersion relation of the two different structures were calculated by tracking the reflectivity minima across different incidence angles [Fig. 3(b)]. The first reflectivity minima (rebound) of the DBR at the stop band edge gives rise to the TP mode [Fig. 3(c)]. A blue shift (from 460 to $454 \mathrm{~nm}$ ) as well as a reduction in linewidth (from 6 to $2 \mathrm{~nm}$ ) of the TP mode is observed with increasing $\mathrm{Ag}$ layer thickness (from 0 to $55 \mathrm{~nm}$ ) [Fig. 3(c)]. Also, the reflectivity values at the TP resonance $(\sim 0.2)$ reaches its minimum at $50 \mathrm{~nm}$ of $\mathrm{Ag}$, confirming the optimal thickness of $\mathrm{Ag}$ for our experiment. Figures 3(d) and 3(e) indicate the tunability of the TP mode by adjusting $n_{p o r}$ and $\rho$, respectively; A red shift of the TP mode is observed by increasing either $n_{p o r}$ or $\rho$, keeping the layer thickness constant. The refractive index contrast between the $\mathrm{GaN}$ and nanoporous $\mathrm{GaN}$ layer is enhanced by increasing $\rho$, which results in a wider stop band, pushing the TP mode to longer wavelengths. One might think that increasing $n_{p o r}$ reduces the stop band width, resulting in a blue shift of the TP mode. However, since the layer thicknesses remain constant $\left(\lambda_{0} / 4 n_{\text {GaN }}\right.$ and $\lambda_{0} / 4 n_{\text {low }}$ for $\rho=53 \%$ ), the central wavelength of the DBR is red shifted with increasing $n_{p o r}$. As a result, the TP mode of the structure is in fact red shifted for higher $n_{\text {por }}$ compared to the air-filled pores $\left(n_{\text {por }}=1\right)$. Figures $3(\mathrm{f})$ and $3(\mathrm{~g})$ show the calculated reflectivity spectra of the TP structure for different values of $n_{p o r}$ at normal incidence and at $45^{\circ}$ incident angle, respectively. The results indicate a significant shift of the TP mode ( 4 to $7 \mathrm{~nm}$ ) when $n_{\text {por }}$ changes from 1 to 1.3 , indicating its potential application in optical sensing. 


\section{Optics EXPRESS}
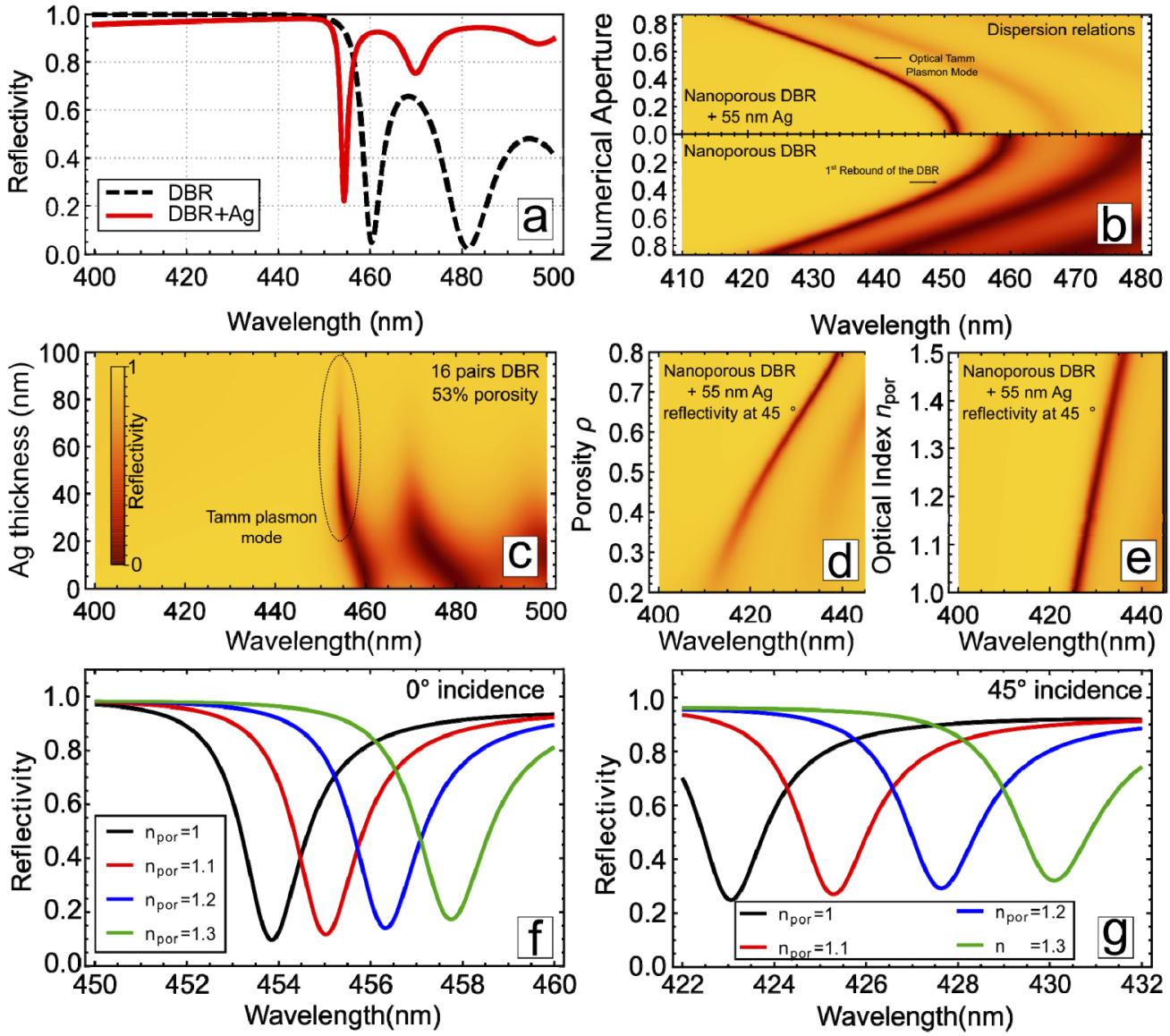

Fig. 3. (a) Simulated reflectivity of a 16 pairs nanoporous GaN DBR (dash black line) and a $55 \mathrm{~nm}$ silver layer on top of the nanoporous GaN DBR (solid red line). (b) TM simulated dispersion relation of TP mode (top) and a DBR (bottom). (c) Calculated reflectivity spectra of TP structure as a function of the thickness of the Ag layer. (d) Calculated reflectivity spectra of TP structure at $45^{\circ}$ incidence angle as a function of (d) $\rho$ and (e) $n_{\text {por }}$ in the nanoporous GaN layer. Reflectivity spectra of a nanoporous TP structures for different $n_{\text {por }}$ values (corresponding to different solvents in the pores) at (f) normal and (g) 45 degree of incidence.

\section{Experimental details}

To experimentally verify the simulation results, a TP structure composed of a $55 \mathrm{~nm} \mathrm{Ag}$ layer (designed based on the optimum $\mathrm{Ag}$ thickness from simulation result of Fig. 3(c)) on top of a nanoporous GaN DBR was fabricated. The DBR sample was grown on a semipolar (20. $\overline{21})$ bulk GaN substrate from Mitsubishi Chemical Corporation (MCC). Semipolar (201) plane was chosen due to its advantages over all other GaN crystal planes for optoelectronic applications. The advantages of this plane compared to other planes include large wavefunction overlap and large radiative recombination rates [27], high-indium incorporation efficiency [28], and high material gain [29]. The sample was grown using an atmospheric vertical design metalorganic chemical vapor deposition (MOCVD), where trimethylgallium (TMGa), $\mathrm{NH}_{3}$, and $\mathrm{SiH}_{4}$ were used as sources of elemental $\mathrm{Ga}, \mathrm{N}$, and Si dopants, respectively. A $300 \mathrm{~nm}$-thick unintentionally doped (UID) GaN buffer layer was first grown on the substrate to improve the morphology. Then, 


\section{Optics EXPRESS}

16 pairs of alternating doped and UID GaN layers were grown with the designed thicknesses of $60 \mathrm{~nm}$ and $45 \mathrm{~nm}$, respectively to target the desired stop-band wavelengths. The doping level for the $n$-type layer was [Si]: $5.5 \times 10^{19} \mathrm{~cm}^{-3}$, as verified by secondary-ion mass spectroscopy (SIMS). All layers were grown at $1180^{\circ} \mathrm{C}$ (thermocouple temperature) and atmospheric pressure.

The DBR was then formed using a wet EC etching technique, as schematically described in Fig. 4. Rectangular trenches were etched down to the substrate using standard photolithography and a $\mathrm{Cl}_{2}$-based reactive ion etching (RIE) opening up a window to the highly doped layers. An indium metal contact was rubbed on the backside of the sample and held with conductive forceps, partially submerging the sample in an oxalic acid bath while keeping the metal contact away from the solution [Fig. 4(a)]. The highly doped layers were then porosified by applying a voltage through a submerged $\mathrm{Pt}$ mesh cathode at $4 \mathrm{~V}$ for 2 hours and $200 \mathrm{rpm}$ stirring. A strong dependence of the EC porosification process on the bias voltage and the doping level is expected [20,21], as schematically shown in Fig. 4(b). The porous GaN area was later verified to undercut $30 \mu \mathrm{m}$ in from the trench edges at approximately $50 \%$ porosity determined by focused ion beam (FIB) cross-sectional and SEM imaging. Figure 5 shows a high-resolution cross-sectional scanning electron microscopy (SEM) image of the processed DBR after EC etching. According to the SEM image of Fig. 5, the size and distribution uniformity of the pores for semipolar (2021) (Fig. 5) is very similar to what has been reported for $c$-plane [21] and $m$-plane [20]. All the doped layers were uniformly porosified resulting in pore sizes $\sim 30-50 \mathrm{~nm}$. A porosity of $\sim 50 \%$ was realized from evaluation of the SEM image of Fig. 5 using an approach similar to what is shown in Ref. [20]. A refractive index of $n_{\text {low }} \sim 1.83$ was realized for the porous layer by the
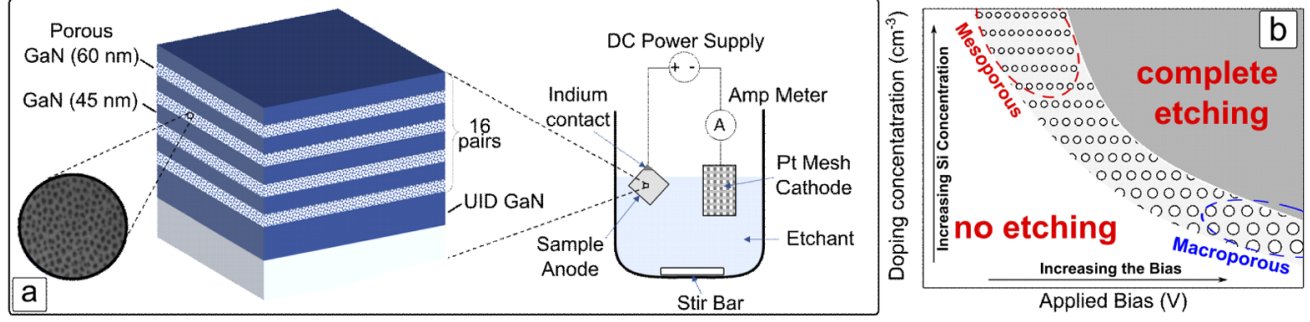

Fig. 4. (a) Schematics of an EC etching setup used to porosify the doped GaN layers and form the nanoporous GaN DBRs. (b) Schematic representation of different etching regimes in the EC etching of GaN, indicating the strong effect of bias voltage and doping concentration on the porosity of the nanoporous $\mathrm{GaN}$ (from no etching regime to mesoporous/macroporous, and to complete etching regime).

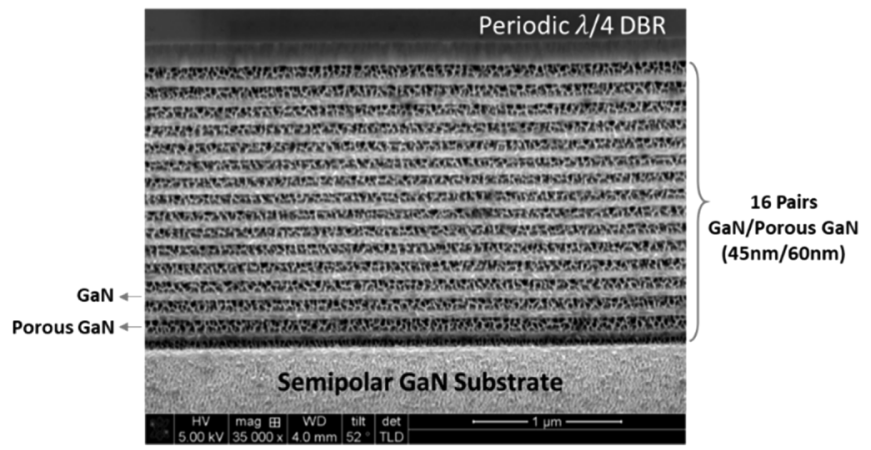

Fig. 5. High-resolution SEM image of a 16-pair nanoporous GaN DBR processed using the EC etching method. 
VAT, as described above [Eq. (1)]. Reflectance spectra of the structure were recorded using a commercial Thin-Film UV-Vis spectrometer (Filmetrics F10-RT-UVX). A $55 \mathrm{~nm}$ Ag layer was then blanket deposited on the DBR sample using an electron-beam evaporator.

\section{Experimental results and discussion}

Figure 6(a) shows an experimentally measured reflectivity spectrum of the DBRs before and after Ag deposition. According to the plot of Fig. 6(a), the DBR reflectivity spectrum shows a stop-band below $460 \mathrm{~nm}$ [dash black line in Fig. 6(a)]. No sharp reflectivity minima, corresponding to the high wavelength edge of the of the DBR at $\sim 460 \mathrm{~nm}$ from simulations [Fig. 3(a)] is observed experimentally [Fig. 6(a)], which may be due to the potential spatial nonuniformity of the nanoporous DBR within the measurement spot size. However, the absence of the $460 \mathrm{~nm}$ reflectivity minima is not detrimental to the stop-band of the structure [30], required for the existence of the TP mode. The spectrum of the DBR structure is measured at the same spot before and after the Ag blanket deposition. The reflectivity of the TP structure (DBR $+55 \mathrm{~nm}$ $\mathrm{Ag}$ ) shows a steep dip near $450 \mathrm{~nm}$ [solid red line in Fig. 6(a)] indicating a TP mode, $\sim 5 \mathrm{~nm}$ below the first rebound wavelength of the DBR, which is in good agreement with the simulation results of Fig. 3(a). The spectrum of the $\mathrm{Ag}+\mathrm{GaN}$ is also plotted [grey dots in Fig. 6(a)] for comparison.

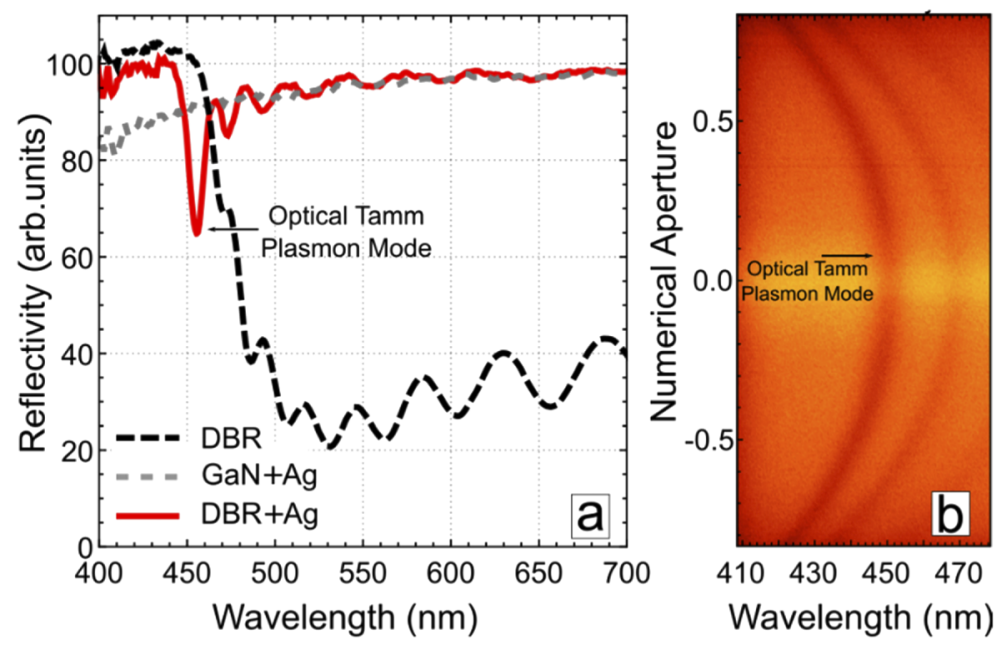

Fig. 6. (a) Measured reflectivity spectra at normal incidence of nanoporous GaN DBR (dash black line), a $55 \mathrm{~nm} \mathrm{Ag} \mathrm{layer} \mathrm{on} \mathrm{top} \mathrm{of} \mathrm{a} 3 \mu \mathrm{m} \mathrm{GaN}$ buffer on GaN substrate (grey dots) and a TP structure with a $55 \mathrm{~nm}$ layer of $\mathrm{Ag}$ on top of a nanoporous GaN DBR (solid red line). (b) Experimental dispersion relation of a $55 \mathrm{~nm}$ layer of $\mathrm{Ag}$ on top of a nanoporous GaN DBR. The parabolic dispersion relation of the TP structure is clearly visible.

To further evaluate the TP mode dispersion, energy-momentum reflectance spectroscopy was used to evaluate the dispersion curve for the structure. Momentum-resolved reflectance spectra were measured using techniques described thoroughly in Ref. [31] and a setup similar to the ones describe in Refs. [32,33]. Figure 6(b) indicates the experimental dispersion relation of TP Plasmon structure in TM polarization. As previously demonstrated, the TP mode exhibits a parabolic dispersion relation, characteristic of the Fabry-Perot like optical mode. The reflectivity spectra with and without metal, as well as the measured dispersion, unambiguously demonstrate the first observation of TP modes in any porous GaN-based systems. The TP mode quality factor is approximately 300, which is lower than those reported earlier [5] ( 1000). Increasing the number of pairs (to obtain 100\% reflectivity) and optimization of the epitaxial growth, porosification, 
deposition condition (i.e. deposition rate, uniformity, and quality), and the uniformity of the DBRs are expected to further enhance the quality factor. Hence, systematic optimizations of the abovementioned parameters to improve the quality factor is a subject of our future investigations. Furthermore, the use of $\mathrm{Ag}$ as the metallic layer may not be an ideal choice particularly for wavelength ranges below $400 \mathrm{~nm}$, as it's approaching the plasmon resonance of $\mathrm{Ag}$ [34,35], which could dramatically decrease the quality factor of the mode. The use of Al [36], $\mathrm{Cr}$ [37] or even graphene [38] instead of Ag could be alternatives to improve the quality factor of the TP on a GaN or AlGaN nanoporous DBR for UV applications. Also, we would be interested in investigating air-gap DBRs (100\% porosity) [39,40], since they will provide much larger refractive index contrast and higher quality factor as well as higher optical sensitivity. Moreover, DBR-side TP structures can also be investigated to further improve the quality factor, as previous reports [41-43] in other material systems indicate higher quality factors in DBR-side TP structures compared to metal-side TPs. However, the implementation of DBR-side TP structures could be challenging for metal/nanoporous GaN systems due to the need for the epitaxy of $\mathrm{GaN}$ on metal and may require special techniques such as epitaxial lateral overgrowth (ELO) [44]. Finally, we want to stress the broad range of wavelength achievable with the nanoporous GaN DBR, from near UV to IR. Nanoporous AlGaN DBR systems can also be considered for application in the deep UV ranges.

\section{Conclusion}

In summary, we demonstrate a TP mode in a metal-DBR structure using a nanoporous GaN DBR system. A strong dependence of the TP mode position on the nanoporous GaN DBR properties was observed based on calculations (shift of $\sim 4-7 \mathrm{~nm}$ when $n_{\text {por }}$ changes from 1.0 to 1.3), indicating potentials for optical sensing applications. An optimum thickness of $\mathrm{Ag}(\sim 55$ $\mathrm{nm}$ ) was realized from theoretical calculations and was deposited on the DBRs. The DBR +55 $\mathrm{nm} \mathrm{Ag}$ structure shows a dip in reflectivity at $\sim 454 \mathrm{~nm}$ corresponding to a TP mode. The results from mode dispersion with energy-momentum reflectance spectroscopy measurements also support the presence of a TP mode at the metal-nanoporous GaN DBR interface. The TP on lattice-matched nanoporous GaN DBRs have applications in active and passive optoelectronics, including optical sensors, resonant cavity LEDs, and lasers.

\section{Funding}

Solid State Lighting and Energy Electronics Center, University of California Santa Barbara (100010947); National Science Foundation (DMS-1839077); Simons Foundation (601952, JS, 601954, CW); Office of Naval Research (N00014-19-1-2004).

\section{Acknowledgments}

A portion of this work was performed at the UCSB nanofabrication facility.

\section{Disclosures}

The authors declare no conflicts of interest.

\section{References}

1. A. V. Kavokin, I. A. Shelykh, and G. Malpuech, "Lossless interface modes at the boundary between two periodic dielectric structures," Phys. Rev. B 72(23), 233102 (2005).

2. M. E. Sasin, R. P. Seisyan, M. A. Kalitteevski, S. Brand, R. A. Abram, J. M. Chamberlain, A. Y. Egorov, A. P. Vasil'Ev, V. S. Mikhrin, and A. V. Kavokin, “Tamm plasmon polaritons: Slow and spatially compact light,” Appl. Phys. Lett. 92(25), 251112 (2008).

3. A. P. Vinogradov, A. V. Dorofeenko, S. G. Erokhin, M. Inoue, A. A. Lisyansky, A. M. Merzlikin, and A. B. Granovsky, "Surface state peculiarities in one-dimensional photonic crystal interfaces," Phys. Rev. B 74(4), 045128 (2006). 
4. M. Kaliteevski, I. Iorsh, S. Brand, R. A. Abram, J. M. Chamberlain, A. V. Kavokin, and I. A. Shelykh, "Tamm plasmon-polaritons: Possible electromagnetic states at the interface of a metal and a dielectric Bragg mirror," Phys. Rev. B 76(16), 165415 (2007).

5. C. Symonds, S. Azzini, G. Lheureux, A. Piednoir, J. M. Benoit, A. Lemaitre, P. Senellart, and J. Bellessa, "High quality factor confined Tamm modes," Sci. Rep. 7(1), 3859 (2017).

6. S. Azzini, G. Lheureux, C. Symonds, J.-M. Benoit, P. Senellart, A. Lemaitre, J.-J. Greffet, C. Blanchard, C. Sauvan, and J. Bellessa, "Generation and Spatial Control of Hybrid Tamm Plasmon/Surface Plasmon Modes," ACS Photonics 3(10), 1776-1781 (2016).

7. C. Symonds, A. Lemaître, P. Senellart, M. H. Jomaa, S. Aberra Guebrou, E. Homeyer, G. Brucoli, and J. Bellessa, "Lasing in a hybrid GaAs/silver Tamm structure," Appl. Phys. Lett. 100(12), 121122 (2012).

8. R. Brückner, A. A. Zakhidov, R. Scholz, M. Sudzius, S. I. Hintschich, H. Fröb, V. G. Lyssenko, and K. Leo, "Phase-locked coherent modes in a patterned metal-organic microcavity," Nat. Photonics 6(5), 322-326 (2012).

9. C. Symonds, G. Lheureux, J. P. Hugonin, J. J. Greffet, J. Laverdant, G. Brucoli, A. Lemaitre, P. Senellart, and J. Bellessa, "Confined Tamm Plasmon Lasers," Nano Lett. 13(7), 3179-3184 (2013).

10. O. Gazzano, S. Michaelis de Vasconcellos, K. Gauthron, C. Symonds, P. Voisin, J. Bellessa, A. Lemaître, and P. Senellart, "Single photon source using confined Tamm plasmon modes," Appl. Phys. Lett. 100(23), 232111 (2012).

11. B. Auguié, M. C. Fuertes, P. C. Angelomé, N. L. Abdala, G. J. A. A. Soler Illia, and A. Fainstein, "Tamm Plasmon Resonance in Mesoporous Multilayers: Toward a Sensing Application," ACS Photonics 1(9), 775-780 (2014).

12. O. Gazzano, S. M. de Vasconcellos, K. Gauthron, C. Symonds, J. Bloch, P. Voisin, J. Bellessa, A. Lemaître, and P. Senellart, "Evidence for Confined Tamm Plasmon Modes under Metallic Microdisks and Application to the Control of Spontaneous Optical Emission,” Phys. Rev. Lett. 107(24), 247402 (2011).

13. G. Lheureux, S. Azzini, C. Symonds, P. Senellart, A. Lemaître, C. Sauvan, J.-P. Hugonin, J.-J. Greffet, and J. Bellessa, "Polarization-Controlled Confined Tamm Plasmon Lasers," ACS Photonics 2(7), 842-848 (2015).

14. Y. Zheng, F. Xiao, W. Liu, and X. Hu, "Purcell effect and light extraction of Tamm-plasmon-cavity green light-emitting diodes," Opt. Express 27(21), 30852 (2019).

15. T. Tawara, H. Gotoh, T. Akasaka, N. Kobayashi, and T. Saitoh, "Low-threshold lasing of InGaN vertical-cavity surface-emitting lasers with dielectric distributed Bragg reflectors," Appl. Phys. Lett. 83(5), 830-832 (2003).

16. H. M. Ng, T. D. Moustakas, and S. N. G. Chu, "High reflectivity and broad bandwidth AlN/GaN distributed Bragg reflectors grown by molecular-beam epitaxy," Appl. Phys. Lett. 76(20), 2818-2820 (2000).

17. J.-F. Carlin and M. Ilegems, "High-quality AlInN for high index contrast Bragg mirrors lattice matched to GaN," Appl. Phys. Lett. 83(4), 668-670 (2003).

18. M. Kuramoto, S. Kobayashi, T. Akagi, K. Tazawa, K. Tanaka, T. Saito, and T. Takeuchi, "High-output-power and high-temperature operation of blue GaN-based vertical-cavity surface-emitting laser," Appl. Phys. Express 11(11), 112101 (2018).

19. K. Ikeyama, Y. Kozuka, K. Matsui, S. Yoshida, T. Akagi, Y. Akatsuka, N. Koide, T. Takeuchi, S. Kamiyama, M. Iwaya, and I. Akasaki, "Room-temperature continuous-wave operation of GaN-based vertical-cavity surface-emitting lasers with n-type conducting AlInN/GaN distributed Bragg reflectors," Appl. Phys. Express 9(10), 102101 (2016).

20. S. Mishkat-Ul-Masabih, T. S. Luk, A. Rishinaramangalam, M. Monavarian, M. Nami, and D. Feezell, "Nanoporous distributed Bragg reflectors on free-standing nonpolar m-plane GaN," Appl. Phys. Lett. 112(4), 041109 (2018).

21. C. Zhang, S. H. Park, D. Chen, D.-W. Lin, W. Xiong, H.-C. Kuo, C.-F. Lin, H. Cao, and J. Han, "Mesoporous GaN for Photonic Engineering - Highly Reflective GaN Mirrors as an Example," ACS Photonics 2(7), 980-986 (2015).

22. S. M. Mishkat-Ul-Masabih, A. A. Aragon, M. Monavarian, T. S. Luk, and D. F. Feezell, "Electrically injected nonpolar GaN-based VCSELs with lattice-matched nanoporous distributed Bragg reflector mirrors," Appl. Phys. Express 12(3), 036504 (2019).

23. R. Anderson, D. Cohen, S. Mehari, S. Nakamura, and S. DenBaars, "Electrical injection of a $440 \mathrm{~nm}$ InGaN laser with lateral confinement by nanoporous-GaN," Opt. Express 27(16), 22764-22769 (2019).

24. S. M. Mishkat-Ul-Masabih, T. S. Luk, M. Monavarian, and D. F. Feezell, "Polarization-pinned emission of a continuous-wave optically pumped nonpolar GaN-based VCSEL using nanoporous distributed Bragg reflectors," Opt. Express 27(7), 9495-9501 (2019).

25. P. B. Johnson and R. W. Christy, "Optical Constant of the Nobel Metals," Phys. Rev. B: Solid State 6(12), 4370-4379 (1972).

26. G. Yu, G. Wang, H. Ishikawa, M. Umeno, T. Soga, T. Egawa, J. Watanabe, and T. Jimbo, "Optical properties of wurtzite structure $\mathrm{GaN}$ on sapphire around fundamental absorption edge $(0.78-4.77 \mathrm{eV})$ by spectroscopic ellipsometry and the optical transmission method," Appl. Phys. Lett. 70(24), 3209-3211 (1997).

27. M. Monavarian, A. Rashidi, A. Aragon, S. H. Oh, M. Nami, S. P. DenBaars, and D. Feezell, "Explanation of low efficiency droop in semipolar $\left(202^{-} 1^{-}\right)$InGaN/GaN LEDs through evaluation of carrier recombination coefficients," Opt. Express 25(16), 19343 (2017).

28. Y. Zhao, Q. Yan, C.-Y. Huang, S.-C. Huang, P. Shan Hsu, S. Tanaka, C.-C. Pan, Y. Kawaguchi, K. Fujito, C. G. Van de Walle, J. S. Speck, S. P. DenBaars, S. Nakamura, and D. Feezell, "Indium incorporation and emission properties of nonpolar and semipolar InGaN quantum wells," Appl. Phys. Lett. 100(20), 201108 (2012).

29. M. Monavarian, A. Rashidi, and D. Feezell, "A Decade of Nonpolar and Semipolar III-Nitrides: A Review of Successes and Challenges,” Phys. Status Solidi A 216(1), 1800628 (2018). 


\section{Optics EXPRESS}

30. L. Pavesi and P. Dubos, "Random porous silicon multilayers: application to distributed Bragg reflectors and interferential Fabry - Pérot filters," Semicond. Sci. Technol. 12(5), 570-575 (1997).

31. R. A. DeCrescent, N. R. Venkatesan, C. J. Dahlman, R. M. Kennard, M. L. Chabinyc, and J. A. Schuller, "Optical Constants and Effective-Medium Origins of Large Optical Anisotropies in Layered Hybrid Organic/Inorganic Perovskites," ACS Nano 13(9), 10745-10753 (2019).

32. J. A. Schuller, S. Karaveli, T. Schiros, K. He, S. Yang, I. Kymissis, J. Shan, and R. Zia, "Orientation of luminescent excitons in layered nanomaterials," Nat. Nanotechnol. 8(4), 271-276 (2013).

33. H. M. Doeleman, F. Monticone, W. den Hollander, A. Alù, and A. F. Koenderink, "Experimental observation of a polarization vortex at an optical bound state in the continuum," Nat. Photonics 12(7), 397-401 (2018).

34. A. Marini, R. Del Sole, and G. Onida, "First-principles calculation of the plasmon resonance and of the reflectance spectrum of silver in the GW approximation," Phys. Rev. B 66(11), 115101 (2002).

35. H. U. Yang, J. D’Archangel, M. L. Sundheimer, E. Tucker, G. D. Boreman, and M. B. Raschke, "Optical dielectric function of silver," Phys. Rev. B 91(23), 235137 (2015).

36. K. M. McPeak, S. V. Jayanti, S. J. P. Kress, S. Meyer, S. Iotti, A. Rossinelli, and D. J. Norris, "Plasmonic Films Can Easily Be Better: Rules and Recipes," ACS Photonics 2(3), 326-333 (2015).

37. A. M. Vyunishev, R. G. Bikbaev, S. E. Svyakhovskiy, I. V. Timofeev, P. S. Pankin, S. A. Evlashin, S. Y. Vetrov, S. A. Myslivets, and V. G. Arkhipkin, "Broadband Tamm plasmon polariton,” J. Opt. Soc. Am. B 36(8), 2299 (2019).

38. H. Lu, X. Gan, B. Jia, D. Mao, and J. Zhao, "Tunable high-efficiency light absorption of monolayer graphene via Tamm plasmon polaritons," Opt. Lett. 41(20), 4743-4746 (2016).

39. R. Sharma, E. D. Haberer, C. Meier, E. L. Hu, and S. Nakamura, "Vertically oriented GaN-based air-gap distributed Bragg reflector structure fabricated using band-gap-selective photoelectrochemical etching," Appl. Phys. Lett. 87(5), 051107 (2005).

40. R. Sharma, Y.-S. Choi, C.-F. Wang, A. David, C. Weisbuch, S. Nakamura, and E. L. Hu, "Gallium-nitride-based microcavity light-emitting diodes with air-gap distributed Bragg reflectors," Appl. Phys. Lett. 91(21), 211108 (2007).

41. B. Auguié, A. Bruchhausen, and A. Fainstein, "Critical coupling to Tamm plasmons," J. Opt. 17(3), 035003 (2015)

42. V. V. Klimov, I. V. Treshin, A. S. Shalin, P. N. Melentiev, A. A. Kuzin, A. E. Afanasiev, and V. I. Balykin, "Optical Tamm state and giant asymmetry of light transmission through an array of nanoholes," Phys. Rev. A 92(6), 063842 (2015).

43. Z.-Y. Yang, S. Ishii, T. Yokoyama, T. D. Dao, M.-G. Sun, P. S. Pankin, I. V. Timofeev, T. Nagao, and K.-P. Chen, "Narrowband Wavelength Selective Thermal Emitters by Confined Tamm Plasmon Polaritons," ACS Photonics 4(9), 2212-2219 (2017).

44. S. Izumi, N. Fuutagawa, T. Hamaguchi, M. Murayama, M. Kuramoto, and H. Narui, "Room-temperature continuouswave operation of GaN-based vertical-cavity surface-emitting lasers fabricated using epitaxial lateral overgrowth," Appl. Phys. Express 8(6), 062702 (2015). 